\title{
EDITORIAL: MEMORIAL ISSUE FOR CHARLES STEIN
}

\author{
By RichaRd J. SAMWORTH ${ }^{1}$ AND Ming YUAN ${ }^{2}$ \\ ${ }^{1}$ Statistical Laboratory, Centre for Mathematical Sciences, Wilberforce Road, Cambridge, CB3 OWB, United Kingdom \\ r.samworth@statslab.cam.ac.uk \\ ${ }^{2} 1255$ Amsterdam Avenue, New York, NY 10027, United States of America \\ ming.yuan@columbia.edu
}

The Institute of Mathematical Statistics (IMS) Council approved a proposal from its Committee on Memorials to dedicate this issue of the Annals of Statistics to Charles M. Stein, who died in 2016 aged 96. This memorialisation is a reflection of Stein's distinction as a mathematical statistician, whose work continues to have a profound impact on the discipline.

As co-editors, we have solicited four articles on some of the most remarkable and enduring of Stein's contributions. Eaton and George (2021) describe the earliest of these, namely Stein's work with Gil Hunt on understanding the relationship between invariant statistical procedures and minimaxity. It was already understood that many statistical problems possess invariance properties with respect to particular groups of transformations, and in such circumstances, it is natural to consider statistical procedures that respect this invariance. As Eaton and George explain, Hunt and Stein set out to understand when the minimax risk over all procedures matches the minimax risk over invariant ones. They cover not only the elegant solution as described by the Hunt-Stein theorem, but also the story of the lost manuscript and the way in which the ideas did finally come to be known.

Strawderman (2021) discusses Stein's seminal result on the inadmissibility of the usual estimator of a multivariate normal mean in three or more dimensions (Stein, 1956b). Invariance played a significant role in Stein's thinking here too, in that a spherically symmetric estimator that is admissible with respect to the set of all spherically symmetric estimators turns out also to be admissible among all estimators. Strawderman describes the way in which the 1956 paper became a launchpad for many further investigations into the Stein shrinkage phenomenon and (in)admissibility. Less directly, the idea of using shrinkage to reduce variance (at the expense of introducing some bias) lies at the heart of ridge regression and other penalised likelihood techniques that have become so popular in recent years.

Within the same volume of the famous Proceedings of the Third Berkeley Symposium on Mathematical Statistics and Probability, Stein (1956a) laid the foundations for the theory of efficient semiparametric inference, as explained by van der Vaart and Wellner (2021). The authors describe Stein's insight that when a parameter space is infinite-dimensional (nonparametric), there may be a one-dimensional least favourable subproblem that is as difficult as the original problem of estimating the underlying parameter. This facilitates an extension of the asymptotic theory of estimation in regular parametric models to one for smooth functionals in infinite-dimensional models. Among several examples and extensions, van der Vaart and Wellner present Stein's classical example of estimating the population median in a location family generated by a symmetric density, showing that absence of knowledge of this symmetric density does not lead to an asymptotic deterioration in the estimation of this median.

Lastly, Chen (2021) outlines Stein's method for normal approximation, beginning with his characterisation of the standard normal distribution in terms of the vanishing of the expectations of certain test functions. This turns out to spawn a powerful technique for bounding a discrepancy between the distribution of a given random variable and that of a standard normal random variable. Chen includes several anecdotes that give an insight into the way Stein's

1

imsart-aos ver. 2020/01/20 file: SteinEditorial.tex date: April 5, 2021 
creative mind worked, such as how Stein discovered this approach when trying to derive a new proof of the central limit theorem for a nonparametric statistics course he was teaching.

In addition to the four articles described above, this issue contains several regular Annals of Statistics papers; all of the authors were happy for their work to appear in this special issue.

Personal reflections. RJS writes: Stein's work had a significant influence on my decision to become a statistician. During my masters year, I wrote an essay on 'Shrinkage estimators', set by Alastair Young; like many others, I was astonished by his inadmissibility result and the beauty of his arguments. All these years later, I remain enchanted by this area. Both of my first two papers (Samworth, 2003, 2005) were concerned with different aspects of James-Stein estimation, namely bootstrap consistency and the construction of confidence sets centred at the positive-part James-Stein estimator.

I was fortunate enough to meet Stein once, as a masters student, when he gave a seminar in King's College, Cambridge. As I recall, there were only a handful of people in the audience, but his sharp intellect and gentle nature nevertheless left a lasting impression on me.

\section{REFERENCES}

Chen, L. (2021) Stein's method of normal approximation: Some recollections and reflections. Ann. Statist., this issue.

Eaton, M. L. and George, E. I. (2021) Charles Stein and invariance: Beginning with the Hunt-Stein theorem. Ann. Statist., this issue.

Samworth, R. (2003) A note on methods of restoring consistency to the bootstrap. Biometrika, 90, 985-990.

Samworth, R. (2005) Small confidence sets for the mean of a spherically symmetric distribution. J. Roy. Statist. Soc., Ser. B, 67, 343-361.

Stein, C. (1956a) Efficient nonparametric testing and estimation. Proceedings of the Third Berkeley Symposium on Mathematical Statistics and Probability, 1954-1955, vol. I, 187-195. University of California Press, Berkeley and Los Angeles.

Stein, C. (1956b) Inadmissibility of the usual estimator for the mean of a multivariate normal distribution. Proceedings of the Third Berkeley Symposium on Mathematical Statistics and Probability, 1954-1955, vol. I, 197-206. University of California Press, Berkeley and Los Angeles.

Strawderman, W. E. (2021) On Charles Stein's contributions to (in)admissibility. Ann. Statist., this issue.

van der Vaart, A. W. and Wellner, J. A. (2021) Stein 1956: Efficient nonparametric testing and estimation. Ann. Statist., this issue. 


\section{PUBLISHED WORKS OF CHARLES M. STEIN}

\section{REFERENCES}

Anderson, T. W., Stein, C. and Zaman, A. (1985) Best invariant estimation of a direction parameter. Ann. Statist., 13, 526-533.

Baldi, P., Rinott, Y. and Stein, C. (1989) A normal approximation for the number of local maxima of a random function on a graph. Probability, statistics, and mathematics, 59-81, Academic Press, Boston, MA.

Diaconis, P. and Stein, C. (1978) Some Tauberian theorems related to coin tossing. Ann. Probab., 6, 483-490.

Efron, B. and Stein, C. (1981) The jackknife estimate of variance. Ann. Statist., 9, 586-596.

Giri, N., Kiefer, J. and Stein, C. (1963) Minimax character of Hotelling's $T^{2}$ test in the simplest case. Ann. Math. Statist., 34, 1524-1535.

James, W. and Stein, C. (1961) Estimation with quadratic loss. 1961 Proc. 4th Berkeley Sympos. Math. Statist. and Prob., Vol. I, 361-379. Univ. California Press, Berkeley, California.

Lehmann, E. L. and Stein, C. (1948) Most powerful tests of composite hypotheses. I. Normal distributions. Ann. Math. Statist., 19, 495-516.

Lehmann, E. L. and Stein, C. (1949) On the theory of some nonparametric hypotheses. Ann. Math. Statist., 20, 28-45.

Lehmann, E. L. and Stein, C. (1950) Completeness in the sequential case. Ann. Math. Statist., 21, 376-385.

Lehmann, E. L. and Stein, C. M. (1953) The admissibility of certain invariant statistical tests involving a translation parameter. Ann. Math. Statist., 24, 473-479.

Stein, C. (1945) A two-sample test for a linear hypothesis whose power is independent of the variance. Ann. Math. Statist., 16, 243-258.

Stein, C. (1946) A note on cumulative sums. Ann. Math. Statist., 17, 498-499.

Stein, C. (1950) Unbiased estimates with minimum variance. Ann. Math. Statist., 21, 406-415.

Stein, C. M. (1951) A property of some tests of composite hypotheses. Ann. Math. Statist., 22, 475-476.

Stein, C. M. (1953) A Two-sample test for a linear hypothesis having power independent of the variance. Ph.D. thesis, Columbia University.

Stein, C. (1955) A necessary and sufficient condition for admissibility. Ann. Math. Statist., 26, 518-522.

Stein, C. (1956a) Efficient nonparametric testing and estimation. Proceedings of the Third Berkeley Symposium on Mathematical Statistics and Probability, 1954-1955, vol. I, 187-195. University of California Press, Berkeley and Los Angeles.

Stein, C. (1956b) Inadmissibility of the usual estimator for the mean of a multivariate normal distribution. Proceedings of the Third Berkeley Symposium on Mathematical Statistics and Probability, 1954-1955, vol. I, 197-206. University of California Press, Berkeley and Los Angeles.

Stein C. (1956c) The admissibility of Hotelling's $T^{2}$-test. Ann. Math. Statist., 27, 616âĂŞ623.

Stein, C. (1959a) An example of wide discrepancy between fiducial and confidence intervals. Ann. Math. Statist., 30, 877-880.

Stein, C. (1959b) The admissibility of Pitman's estimator of a single location parameter. Ann. Math. Statist., 30, 970-979.

Stein, C. (1960) Multiple regression. 1960 Contributions to probability and statistics, 424-443. Stanford Univ. Press, Stanford, Calif.

Stein, C. (1962a) A remark on the likelihood principle. J. Roy. Statist. Soc. Ser. A, 125, 565-568.

Stein, C. M. (1962b) Confidence sets for the mean of a multivariate normal distribution. J. Roy. Statist. Soc. Ser. $B, 24,265-296$.

Stein, C. (1964) Inadmissibility of the usual estimator for the variance of a normal distribution with unknown mean. Ann. Inst. Statist. Math., 16, 155-160.

Stein, C. (1965) Approximation of improper prior measures by prior probability measures. 1965 Proc. Internat. Res. Sem., Statist. Lab., Univ. California, Berkeley, Calif., 1963, 217-240, Springer-Verlag, New York.

Stein, C. (1966) An approach to the recovery of inter-block information in balanced incomplete block designs. 1966 Research Papers in Statistics (Festschrift J. Neyman), 351-366 John Wiley, London.

Stein, C. M. (1968) Some recent developments in mathematical statistics. Proc. Internat. Congr. Math. (Moscow, 1966), 140-157.

Portnoy, S. and Stein, C. (1971) Inadmissibility of the best invariant test in three or more dimensions. Ann. Math. Statist., 42, 799-801.

Stein, C. (1972) A bound for the error in the normal approximation to the distribution of a sum of dependent random variables. Proceedings of the Sixth Berkeley Symposium on Mathematical Statistics and Probability, Vol. II: Probability theory, 583-602. Univ. California Press, Berkeley, Calif., 1972.

Stein, C. (1974) Estimation of the mean of a multivariate normal distribution. Proceedings of the Prague Symposium on Asymptotic Statistics (Charles Univ., Prague, 1973), Vol. II, 345-381.

imsart-aos ver. 2020/01/20 file: SteinEditorial.tex date: April 5, 2021 
Stein, C. (1977) Lectures on multivariate estimation theory. (In Russian). Zap. Nauchn. Sem. Leningrad. Otdel. Mat. Inst. Steklov. (LOMI), 74, 4-65.

Stein, C. M. (1978) Asymptotic evaluation of the number of Latin rectangles. J. Combin. Theory Ser. A, 25, 38-49.

Stein, C. M. (1981) Estimation of the mean of a multivariate normal distribution. Ann. Statist., 9, 1135-1151.

Stein, C. M. (1985) On the coverage probability of confidence sets based on a prior distribution. Sequential methods in statistics, 485-514, Banach Center Publ., 16, PWN, Warsaw.

Stein, C. (1986) Approximate Computation of Expectations. Institute of Mathematical Statistics Lecture NotesMonograph Series, 7. Institute of Mathematical Statistics, Hayward, CA.

Stein, C. (1992) A way of using auxiliary randomization. Probability theory (Singapore, 1989), 159-180, de Gruyter, Berlin.

Stein, C, Diaconis, P., Holmes, S. and Reinert, G. (2004) Use of exchangeable pairs in the analysis of simulations. Stein's method: expository lectures and applications, 1-26, IMS Lecture Notes Monogr. Ser., 46, Inst. Math. Statist., Beachwood, OH.

Stein, C. and Wald, A. (1947) Sequential confidence intervals for the mean of a normal distribution with known variance. Ann. Math. Statist., 18, 427-433. 